\title{
Formation and evolution of spiral arms in galaxies orbiting a Virgo-like cluster
}

\author{
Marcin Semczuk $^{1 \star}$ and Ewa L. Lokas ${ }^{1}$ \\ ${ }^{1}$ Nicolaus Copernicus Astronomical Center, \\ Bartycka 18, 00-716 Warsaw, Poland \\ $\star$ email: msemczuk@camk.edu.pl
}

\begin{abstract}
The origin of spiral structure in disks of galaxies remains an open question. One of the theories predicts that two-armed, grand design spiral arms originate from tidal interactions with another body. Using N-body simulations we find that a Milky Way-like galaxy can develop spiral arms due to tidal force from a cluster-size dark matter halo.
\end{abstract}

Keywords. Galaxies, spiral galaxies, spiral arms, N-body simulations, Virgo cluster

\section{Introduction}

We performed three simulations of a Milky Way-like galaxy orbiting a Virgo-like cluster approximated as an NFW dark matter halo (Navarro et al. 1997). The galaxy was an Nbody realisation of an exponential stellar disk embedded in an NFW dark matter halo. It was placed on three prograde, eccentric orbits. The evolution of the system was followed with GADGET-2 N-body code (Springel 2005) for 10 Gyr.

\section{Results}

We find that the spiral arms form for each orbit during the pericenter passage. Arms are found to be grand-design, approximately logarithmic structures but their shape is dynamic and not stable. Initially they are wide tidal structures. However they are bound to the galaxy, so they quickly wind up towards the center of the disk. Later they keep winding up and dissolving until they are triggered again by the next pericenter passage.

We confirm this transient and recurrent behaviour by analyzing the evolution of the pitch angle and the arm strength in time using the expansion in logarithmic spirals (Sellwood \& Athanassoula, 1986). Results of these measurements for the most extended orbit can be found in Semczuk \& Łokas (2015). During the pericenters the pitch angle has values of $30^{\circ}-40^{\circ}$, but it exponentially decreases with time reaching $\sim 5^{\circ}$ after one orbital period. The slope of the time dependence of the pitch angle is steeper for tighter orbits, hence the spiral arms are most persistent for the most extended orbit. However, the measurements of the arm strength reveal that the arms are strongest for the tightest orbit.

\section{References}

Navarro, J. F., Frenk, C. S., \& White, S. D. 1997, ApJ, 490, 493

Springel, V. 2005, MNRAS, 364, 1105

Sellwood, J. A. \& Athanassoula, E. 1986, MNRAS, 221, 195

Semczuk, M. \& Łokas, E. L. 2015, arXiv: 1512.06005 